When insemination occurs early in the menstrual cycle the sperm may be over-ripe, and when fertilisation occurs later the ova may be over-ripe. Both over-ripe sperm and over-ripe ova are associated with an increased incidence of chromosomal abnormalities, blighted ova, and death of the blastocyst, and it has been suggested that in such cases female embryos may have a higher incidence of abnormalities and death. ${ }^{8}$ When early or late insemination results in fertilisation of an over-ripe ovum or by an over-ripe sperm the survival of male embryos may thus be relatively increased, resulting in an increased male to female sex ratio at birth. Similarly, the development of the embryo from an over-ripe sperm or ova may be impaired, resulting in delayed and low implantation of the embryo in the uterus and hence placenta praevia.

Placenta praevia may thus be due to delayed development and implantation of the blastocyst resulting from early or late insemination, which is associated with an increase in the male to female sex ratio. The progressive increase in the sex ratio with increasing parity in women with placenta praevia is difficult to explain unless increasing parity is in some way associated with an increase in the incidence of fertilisation of over-ripe ova or by over-ripe sperms. The significant increase in the sex ratio nevertheless suggests that early or late insemination and impaired development of the embryo may be a predisposing factor in placenta praevia.

\section{References}

1 MacGillivray I. Eclampsia, the hypertensive disease of pregnancy. London, Philadelphia, Toronto: B Saunders, 1983.

Hall M, Carr-Hill $\mathrm{R}$. Impact of sex ratio on onset and management of labour. $\mathrm{Br} \mathrm{Med}$ 1982;285:401-3.

3 Bartholemew DJ. On Chassen's test for order. Biometrics 1963;19:188-91.

4 Kantor $S$, Winkelstein W, Ibrahim MA. A note on the interpretation of the ridit as a quantile rank. Am $\mathcal{Y}$ Epidemiol 1968;87:609-15.

5 Payne C. The log-linear model for contingency table analysis. In: Payne C, O'Muircheartaigh CA, eds. Model fiting. London: Wiley, 1977.

6 Harlap S. Gender of infants conceived on different days of the menstrual cycle. N Engl 7 Med 979;300:1445-8.

7 Guerrero R, Rojas OI. Spontaneous abortion and aging of human ova and spermatozoa. $N$ Engl $\mathcal{J}$ Med 1975;293:573-5.

8 Witschi E. Teratogenic effects of overripeness of the egg. In: Fraser FC, McKusick VA, Robinson R, eds. Congenital malformations. Amsterdam: Excerpta Medica, 1970. (International Congress Series No 204.)

(Accepted 2 December 1985)

\title{
An epidemiological study of the immunogenetic aetiology of pre-eclampsia
}

\author{
BETH W ALDERMAN, RHODA S SPERLING, JANET R DALING
}

\begin{abstract}
A population based case-control study of the association between dissimilar race of parents and risk of pre-eclampsia was undertaken. Data on singleton births in Washington State in 1981 were available for analysis from birth certificates. All mothers recorded as having pre-eclampsia and a sample of mothers who did not have pre-eclampsia were eligible for comparison with regard to racial dissimilarity between parents. Women with previously diagnosed cardiovascular disease and diabetes were excluded. After the confounding effects of maternal parity and race had been controlled for, racial dissimilarity of parents was associated with a 1.9-fold increased risk of pre-eclampsia (95\% confidence interval $=1 \cdot 3-2 \cdot 8$; number of cases $=973$, of controls $=$ 1480).
\end{abstract}

This finding supports the theory that genetic dissimilarity of father and mother has a role in pre-eclampsia and is consistent with an immunogenetic aetiology.

\footnotetext{
Department of Preventive Medicine and Biometrics, University of Colorado Medical School, Denver, Colorado
}

BETH W ALDERMAN, MD, MPH, assistant professor

\footnotetext{
Department of Obstetrics, Gynecology, and Reproductive Medicine, Mount Sinai Hospital, New York

RHODA S SPERLING, MD, clinical instructor
}

Department of Epidemiology, University of Washington School of Public Health, Seattle, Washington State

JANET R DALING, PHD, associate professor

Correspondence to: Dr Beth W Alderman, Department of Preventive Medicine and Biometrics, University of Colorado Health Sciences Center, Campus Box C245, 4200 East Ninth Avenue, Denver, Colorado 80262.

\section{Introduction}

Previous studies have linked paternal factors with pre-eclampsia. ${ }^{12}$ Several reports have indicated that change of paternity is associated with an increased occurrence of pre-eclampsia among multiparous women, ${ }^{3.5}$ while others have suggested that exposure to paternal spermatic antigens may protect against pre-eclampsia. ${ }^{6}$ Such observations support the theory that pre-eclampsia may represent a disorder in the maternal immune response to paternally inherited antigens expressed by the fetus.

Some investigators believe that paternal antigens may increase the risk of pre-eclampsia through genetic dissimilarity from the mother, which results in "immunological" incompatibility between fetus and mother. ${ }^{7}$ This theory is supported by a large epidemiological study of a highly consanguineous population, which found that paternal unrelatedness to the mother was associated with an increased risk of pre-eclampsia. ${ }^{8}$ Other investigators, however, believe that the depression of the maternal immune response in pre-eclampsia may result from inadequate stimulation of the immune response owing to genetic similarity of the father and mother. ${ }^{9}$

We designed a population based case-control study to re-examine the relation between pre-eclampsia and a global measure of genetic dissimilarity of mother and father. Dissimilar race of parents, rather than lack of consanguinity, was used as the indicator of genetic dissimilarity.

\section{Methods}

Birth certificates for all single births that occurred in Washington State in 1981 were used to identify subjects and provide data for analysis. All women who were recorded as having pre-eclampsia on the birth certificate were eligible for inclusion in the study. According to protocol, the birth certificate is completed within two days of birth by a nurse or doctor who was present at the birth or by a member of staff at the hospital who has been trained by the state health department. If the attending doctor has diagnosed pre-eclampsia a box labelled pre-eclampsia is to be ticked on the certificate. This protocol is followed closely, as shown by a recent study, which found that after the 
checklist format was instituted in 1980 the number of cases of pre-eclampsia identified on birth certificates reached $97 \%$ of the number identified by hospital survey. ${ }^{10}$

This method probably identifies patients with severe pre-eclampsia, as diagnosed by the Nelson criteria, as pre-eclampsia is generally defined in the United States as hypertension accompanied by proteinuria or oedema. " The diagnostic criteria of the American College of Obstetrics and Gynecology for pre-eclampsia include the development of hypertension induced by pregnancy after the 20th week accompanied by proteinuria or generalised oedema, or both. Hypertension is defined as raised blood pressure on at least two occasions six or more hours apart. Blood pressure is considered to be raised if the diastolic pressure is $\geqslant 90 \mathrm{~mm} \mathrm{Hg}$, the systolic pressure is $\geqslant 140$ $\mathrm{mm} \mathrm{Hg}$, or there is an increase of $\geqslant 30 \mathrm{~mm} \mathrm{Hg}$ in systolic pressure or $\geqslant 15$ $\mathrm{mm} \mathrm{Hg}$ in diastolic pressure above the patient's normal levels. ${ }^{11}$ These criteria yield lower estimates of the incidence of pre-eclampsia than British criteria as they exclude women with isolated hypertension or proteinuria. ${ }^{12}$

A systematic sample of women who did not have pre-eclampsia was selected for comparison (controls). The sample was chosen by selecting every 35 th record from a file of all birth certificates on which the mother had not been recorded as having pre-eclampsia. The file was ordered by county and date of birth and was irregularly periodic with regard to these two factors. Because of the irregularity this system should have yielded an essentially random sample. ${ }^{13}$

To limit the study as much as possible to hypertension induced by pregnancy rather than pre-existing hypertension pre-eclamptic and non-preeclamptic women who were recorded on the birth certificate as having a history of hypertension, heart disease, chronic renal failure, or diabetes were excluded from the analysis.

Maternal and paternal race were both required for assignment of the infant's race and were both recorded on the birth certificate. ${ }^{14}$ Data on race were available in these categories: Mexican or Chicano (Hispanic); Chinese, Japanese, Filipino, Hawaiian, or other Asian and Pacific Islander (Asian); Indian or Red (native American); negro, black, or coloured (black); white or Puerto Rican (white). Maternal and paternal race were considered to be dissimilar when they fell into different categories and similar when they fell into the same category. Evidence from previous work on population analyses of HLA-A, HLA-B, HLA-C, HLA-DR, and other genetic markers indicates that such categories may be used to separate subjects into genetically dissimilar groups. ${ }^{15}$

Other previously reported and possible risk factors for pre-eclampsia for which data were available from birth certificates included maternal age, race, and parity, maternal and paternal occupational state, ${ }^{12}$ marital state,${ }^{16}$ sex of infant,${ }^{17}$ history of miscarriage, ${ }^{18}$ interval since last live birth, and interval since last fetal death. The relation between pre-eclampsia and each of these factors was investigated by categorical analysis, using odds ratios and standardised odds ratios. ${ }^{1920}$ Factors shown by this method to be related to pre-eclampsia were considered to be potential confounders of the relation between pre-eclampsia and dissimilar race of parents. ${ }^{21}$

The relative risk of pre-eclampsia associated with dissimilar race of parents was estimated by the odds ratio, for which confidence intervals were calculated by the test based method. ${ }^{22}$ The odds ratio was evaluated for confounding by stratification and adjustment for each of the potential confounders by the Mantel-Haenszel method. ${ }^{23}$ If the adjusted odds ratio differed from the unadjusted odds ratio confounding was considered to be present. ${ }^{24}$ For the final analysis the odds ratio was simultaneously stratified and adjusted for all confounders by Mantel-Haenszel adjustment. In addition, each maternal-paternal racial combination was investigated to determine whether the increased risk of pre-eclampsia was limited to any particular subgroup.

\section{Results}

We identified 1404 women with pre-eclampsia, corresponding to a yearly incidence of pre-eclampsia throughout the state of $22 / 1000$ live births. After women with cardiovascular disease, renal disease, and diabetes and those for whom data required for the final analysis were lacking had been excluded there were 973 women with pre-eclampsia and 1480 women without available for analysis (table I)

The unadjusted odds ratio relating dissimilar race of parents to preeclampsia was $1 \cdot 5$, suggesting that there was a $1 \cdot 5$-fold increase in risk of preeclampsia associated with dissimilar race. Maternal age, race, and occupation, marital state, parity, history of spontaneous abortion, interval since the last live birth, interval since the last fetal death, and paternal occupation were associated with pre-eclampsia and were considered to be potential confounders of the odds ratio. Stratification of the odds ratio by each potential confounder showed that Mantel-Haenszel odds ratios adjusted for maternal race, parity, and interval between births differed from 1.5 (table II). Simultaneous stratification by maternal race, parity, and interval between births showed that dissimilar race of parents was associated with a $1 \cdot 9$-fold increased risk of pre-eclampsia (95\% confidence interval $=1 \cdot 3,2 \cdot 8)$. The increased risk was not limited to any particular racial combination of parents.

\section{Discussion}

This study shows that dissimilar race of mother and father is associated with an increased risk of pre-eclampsia. The results also suggest that genetic dissimilarity is an important cause of preeclampsia. Racial dissimilarity of father and mother was associated with $9 \cdot 4 \%$ of the cases of pre-eclampsia in Washington..$^{25}$ The total proportion of pre-eclampsia associated with genetic dissimilarity should be very much larger than that in view of the genetic dissimilarity that exists between parents within racial categories.

The results of the study may have been influenced by the retrospective use of the doctor's diagnosis of pre-eclampsia. Women who did not meet the strict criteria of the American College of Obstetrics and Gynecology for pre-eclampsia may have been included among the cases, and some women who did meet these criteria may have been included among the controls. Both of these errors would have diminished distinctions between the two groups and resulted in underestimation of the true risk of pre-eclampsia associated with dissimilar race of parents.

If, by chance, physicians were more likely to diagnose women as having pre-eclampsia when the mother and father were of different race, the risk could have been overestimated. Two observations suggest that this was unlikely. Firstly, more frequent diagnosis is unlikely as dissimilar race of parents is not generally thought to be related to pre-eclampsia, and, secondly, increased diagnosis through more frequent contact with doctors is unlikely as the month in which prenatal care began was similar in those with and without pre-eclampsia.

Another factor that may have affected the results of the study is the use of differences in racial or ethnic groupings, or both, as global measures of genetic dissimilarity. The Hispanic group, in

TABLE I-Women with pre-eclampsia (cases) and without (controls) included in the analysis ${ }^{\star}$

\begin{tabular}{lcc}
\hline & $\begin{array}{c}\text { No of cases } \\
\text { (\% of those eligible) }\end{array}$ & $\begin{array}{c}\text { No of controls } \\
\text { (\% of those eligible) }\end{array}$ \\
\hline Total identified & 1404 & 1816 \\
No excluded for: & 83 & 4 \\
$\quad$ Hypertension & 4 & 1 \\
Heart disease & 6 & 0 \\
Chronic renal failure & 31 & 3 \\
$\quad$ Diabetes & $1283(100)$ & $1808(100)$ \\
Total eligible & $3(0 \cdot 2)$ & $3(0 \cdot 2)$ \\
Missing data on: & $206(16 \cdot 1)$ & $185(10 \cdot 2)$ \\
$\quad$ Maternal race & $101(7 \cdot 9)$ & $140(7 \cdot 7)$ \\
$\quad$ Paternal race & $973(75 \cdot 8)$ & $1480(81 \cdot 9)$ \\
$\quad$ Parity or birth interval & Total analysed &
\end{tabular}

*Selected from all women who delivered singleton babies in 1981 in Washington State.

TABLE II-Relative risk of pre-eclampsia due to dissimilar race of parents ${ }^{\star}$ stratified and adjusted for other factors

\begin{tabular}{|c|c|c|c|c|}
\hline Factors & Stratum & $\begin{array}{l}\text { No of } \\
\text { cases }\end{array}$ & $\begin{array}{l}\text { No of } \\
\text { controls }\end{array}$ & $\begin{array}{l}\text { Mantel-Haenszel } \\
\text { adjusted relative } \\
\text { risk (95\% confidence } \\
\text { interval) }\end{array}$ \\
\hline Maternal race & $\begin{array}{l}\text { White } \\
\text { Black } \\
\text { Native American } \\
\text { Asian } \\
\text { Hispanic }\end{array}$ & $\begin{array}{r}877 \\
28 \\
16 \\
26 \\
26\end{array}$ & $\begin{array}{r}1328 \\
41 \\
23 \\
57 \\
31\end{array}$ & $1 \cdot 6(1 \cdot 1-2 \cdot 3)$ \\
\hline Parity & $\left\{\begin{array}{c}0 \\
\geqslant 1\end{array}\right.$ & $\begin{array}{l}770 \\
203\end{array}$ & $\begin{array}{l}606 \\
874\end{array}$ & $1 \cdot 8(1 \cdot 1-2 \cdot 5)$ \\
\hline $\begin{array}{l}\text { Birth interval } \\
\text { (years) }\end{array}$ & $\left\{\begin{array}{l}<4 \\
\geqslant 4\end{array}\right.$ & $\begin{array}{r}128 \\
75\end{array}$ & $\begin{array}{l}642 \\
232\end{array}$ & $2 \cdot 0(1 \cdot 3-3 \cdot 4)$ \\
\hline \multicolumn{2}{|c|}{$\begin{array}{l}\text { Race, parity, and } \\
\text { birth interval }\end{array}$} & 973 & 1480 & $1.9(1.3-2 \cdot 8)$ \\
\hline
\end{tabular}


particular, may not be genetically different from North American whites. The Asian category, on the other hand, contains many subgroups that may be genetically distinct, such as Japanese, Laotian, and Pacific Island peoples. The potential effect of these errors in classification (that is, increasing the genetic similarity between the five racial groups or increasing the genetic difference within the groups) would be to obscure a risk due to dissimilar race of parents, not to create a falsely high estimate of the risk.

The use of a global measurement of genetic dissimilarity can overcome some difficulties encountered in immunogenetic studies of pre-eclampsia. Genetic studies of HLA and ABO incompatibility are difficult to interpret, as the loci connected with the aetiology of pre-eclampsia are unknown. Trophoblastic antigens, rather than HLA and ABO antigens, may contribute to the aetiology of preeclampsia. ${ }^{26}$ In addition, such studies are expensive and are restricted by the growing but nevertheless limited understanding of immunogenetic mechanisms. As a result, such studies often compare small groups with regard to many variables, and chance differences may seem significant (unless the analysis is corrected for multiple testing) while important differences may not be detected (owing to lack of statistical power).

The most important potential limitation of the study is that a false increase in the estimate of the risk could have been obtained if dissimilar race of parents were associated with other unidentified risk factors for pre-eclampsia. People who marry across racial, ethnic, and religious boundaries are often older and more likely to have been divorced than people who marry within such groups. ${ }^{27}$ Although we were able to control indirectly for these factors in this study by controlling for age and parity, there may be other nongenetic differences between these groups that account for the increased risk of pre-eclampsia.

On the other hand, the similarity of these results and the results of a previous epidemiological study ${ }^{8}$ would suggest that genetic dissimilarity, rather than other factors, accounted for the increased risk of pre-eclampsia. The previous study was conducted among a highly consanguineous religious group in Turkey, was based in a clinic, and used a low level of consanguinity as a measure of genetic dissimilarity. Our study was conducted in Washington, was population based, and used racial dissimilarity of mother and father as a measure of genetic dissimilarity. It seems unlikely that these two very different studies would have been affected similarly by extraneous factors.

Furthermore, the results of this study support the current theory that pre-eclampsia results from a disorder in the maternal immune response to paternally inherited fetal antigens. Evidence indicates that this disorder may be influenced by many factors, such as maternal inheritance of a predisposition to pre-eclampsia ${ }^{28}$ and maternal history of previous exposure to homologous antigens through blood transfusions ${ }^{30}$ and sexual intercourse. ${ }^{6}$ The antigenic stimulus of the pregnancy is undoubtedly also important, and an excess of antigen-antibody complexes might occur when antigenic stimulus increases because of genetic dissimilarity of the mother and fetus. ${ }^{9}$ Evidence thought to indicate that genetic similarity results in pre-eclampsia is based on reports of disordered maternal immune function and maternal HLA homozygosity. These findings may be more consistent with an inherited maternal immune dysfunction than with genetic similarity, as pointed out by the authors of those reports. ${ }^{931}$ In addition, the lack of evidence for HLA and ABO incompatibility between mother and fetus ${ }^{32}$ does not rule out genetic dissimilarity for reasons discussed above.
In the future the rough concepts of "similarity" and "difference" will, we hope, be refined by the development of more specific hypotheses. Unfortunately, the results of this study do not provide obvious clues for further study. Nevertheless, neither the association between pre-eclampsia and interval between births nor the association between pre-eclampsia and dissimilar race of parents has been noted previously, and both should be studied further.

In conclusion, these results support an association between preeclampsia and genetic dissimilarity of mother and father and the theory that pre-eclampsia has an immunogenetic aetiology.

We thank Dr Richard Berkowitz, Dr Gertrud Berkowitz, and Dr Richard Hamman for their helpful review of this manuscript.

\section{References}

1 Scott JS. Paternal responsibility. Br $\mathcal{Y}$ Obstet Gynaecol 1981;89:785-6.

2 Scott JR, Beer AA. Immunologic aspects of pre-eclampsia. Am $\mathcal{Y}$ Obstet Gynecol 1976;125:418-27. 3 Feeney JG, Scott JS. Pre-eclampsia and changed paternity. Eur 3 Obstet Gynecol Reprod Biol 1980;11:35-8.

4 Ikedife D. Eclampsia in multipara. BrMed J 1980;280:985-6.

5 Chng PK. Occurrence of pre-eclampsia in pregnancies to three husbands: case report. Br 7 Obstet Gynaecol 1982;89:862-3.

6 Marti JJ, Hermann U. Immunogestosis: a new etiologic concept of "essential" EPH gestosis, with special consideration of the primigravid patient. Am $\mathcal{f}$ Obstet Gynecol 1977;128:489-93.

7 Stevenson AC, Say B, Ustaoglu S, Durmus Z. Aspects of pre-eclamptic toxaemia of pregnancy, consanguinity, and twinning in Ankara. $\mathcal{F}$ Med Genet 1976;13:1-8.

8 Stevenson AC, Clare Davison BS, Say B, et al. Contribution of fetal/maternal incompatibility to aetiology of pre-eclamptic toxaemia. Lancet $1971 ;$;i: 1286-9.

9 Scott JS, Jenkins DM, Need JA. Immunology of pre-eclampsia. Lancet 1978;i:704-6.

10 Frost F, Starzyk P, George S, McLaughlin JF. Birth complication reporting: the effect of birth certificate design. Am f Public Health 1984;74:505-6.

11 Pritchard JA, MacDonald PC, Gant NF, eds. Williams obstetrics. 17th ed. Norwalk: AppletonCentury-Crofts, 1985 .

12 Davies AM, Dunlop W. Hypertension in pregnancy. In: Barron SL, Thomas AM, eds. Obstetrical epidemiology. London: Academic Press, 1983:167-208.

13 Kish L. Survey sampling. New York: John Wiley and Sons, 1965.

14 Frost F, Shy K. Racial differences between linked birth and infant death records in Washington state. Am F Public Health 1980;70:974-6.

15 Baur MP, Danilovs JA. Population analysis of HLA-A, B, C, DR, and other genetic markers. In: Terasaki PI, ed. Histocompatibility testing 1980: report of the eighth intermational histocompatibility Terasaki PI, ed. Histocompatibility testing 1980: report of the eighth intermationc
workshop. Los Angeles: UCLA Tissue Typing Laboratory, 1980:955-93.

16 Friedman EA, Neff RK. Pregnancy hypertension: a systematic evaluation of clinical diagnostic criteria. Friedman EA, Neff RK. Pregnancy hypertension: a systematic eralu
Littleton, Massachusetts: PSG Publishing Company, 1977.

17 Chesley LC. Hypertensive disorders in pregnancy. New York: Appleton-Century-Crofts, 1978

18 Shapiro S, Schlesinger ER, Nesbitt REL. Infant, perinatal, maternal, and childhood morality in the United States. Cambridge, Massachusetts: Harvard University Press, 1968.

19 Cornfield J. A method of estimating comparative rates from clinical data. Applications to cancer of the lung, breast, and cervix. Foumal of the National Cancer Institute 1951;11:1269-75.

20 Miettinen OS. Standardization of risk ratios. Am $\mathcal{F}$ Epidemiol 1972;6:383-8.

21 Greenland S, Neutra R. Control of confounding in the assessment of medical technology. Int $\mathcal{J}$ Epidemiol 1980;9:361-7.

22 Miettinen OS. Estimability and estimation in case-referent studies. Am f Epidemiol 1976;103:226 35.

23 Mantel N, Haenszel W. Statistical aspects of the analysis of data from retrospective studies of disease. Foumal of the National Cancer Institute 1959;22:719-48.

24 Dales LG, Ury HK. An improper use of statistical significance testing in studying covariables. Int 7 Epidemiol 1978;7:373-5.

25 Miettinen OS. Proportion of disease caused or prevented by a given exposure, trait, or intervention. Am $\mathcal{X}$ Epidemiol 1974;99:325-32.

26 Sinha D, Wells $M$, Faulk WP. Immunological studies of human placentae: complement components in pre-eclamptic chorionic villi. Clin Exp Immunol 1984;56:175-84

27 Barron $M L$, ed. The blending American. Patterns of intermarriage. Chicago: Quadrangle Books, 1972.

28 Cooper DW, Liston WA. Genetic control of severe pre-eclampsia. 7 Med Genet 1979;16:409-16

29 Sutherland A, Cooper DW, Howie PW, Liston WA, MacGillivray I. The incidence of severe pre eclampsia amongst mothers and mothers-in-law of pre-eclamptics and controls. $\mathrm{Br} \mathcal{F} \mathrm{Obste}$ Gynaecol 1981;88:785-91.

30 Feeney JG, Tovey LAD, Scott JS. Influence of previous blood transfusion on incidence of preeclampsia. Lancet 1977; ;:874-5.

31 Redman CWG, Bodmer JG, Bodmer WF, Beilin LJ, Bonnar J. HLA antigens in severe preeclampsia. Lancet 1978;ii:397-8.

32 Persitz E, Oksenberg J, Amar A, Margalioth EJ, Cohen O, Brautbar C. Histocompatibility antigens, mixed lymphocyte reactivity, and severe pre-eclampsia in Israel. Gynecol Obstet Invest 1983;16:283-91.

(Accepted 13 November 1985) 